\title{
Track Reconstruction with PANDA at FAIR
}

\author{
Michael Papenbrock ${ }^{1, *}$, Walter Ikegami Andersson ${ }^{1,}$, and Jenny Regina ${ }^{1,}$ \\ ${ }^{1}$ Uppsala University
}

\begin{abstract}
The future PANDA experiment at FAIR aims to investigate a wide range of physics processes in antiproton-proton collisions at rates of up to 20 $\mathrm{MHz}$, while employing a purely software-based event filter. To educate the trigger decisions, a full event reconstruction has to be carried out in real time. This challenge is amplified when considering tracks from particles with long lifetimes and displaced decay vertices, which add to the complexity of the reconstruction algorithms. Here, we present modifications to a cellular automatonbased track finder taking detector time-stamps into account in addition to spatial information, as well as several pattern recognition methods for longitudinal track reconstrucion with PANDA's Straw Tube Tracker.
\end{abstract}

\section{Tracking with PANDA}

The future PANDA experiment (Antiproton Annihilation at Darmstadt) [1] opens up a wide range of antiproton induced reactions with a multi-purpose detector (see fig. 1) that has a coverage of nearly $4 \pi$ solid angle. It is currently under construction at the Facility for Antiproton and Ion Research (FAIR) in Darmstadt, Germany. Antiproton beams with high intensity and momenta of up to $15 \mathrm{GeV} / \mathrm{c}$ will be provided by the High Energy Storage Ring (HESR). The event rates are expected to reach up to $20 \mathrm{MHz}$. Due to the nearly continuous beam structure of HESR, events will be distributed evenly in time. A full online reconstruction in real-time is required for PANDA's purely software-based event filter.

The free-streaming data from the detector will be grouped by beam revolutions of HESR, each taking circa $2000 \mathrm{~ns}$. Within these bunches, the reconstruction algorithms not only have to group hits into tracks, but also into events. Therefore, the time-stamps of the hits enter as an additional dimension into the algorithms.

The challenge of real-time reconstruction becomes even harder for physics processes with more complex topologies, i.e. those that involve long-lived particles with secondary decay vertices. Many of the long-established reconstruction algorithms utilise the interaction point as a powerful constraint. Adapting them for tracks that originate several tens of centimetres away from the interaction point can add substantial complexity to these algorithms, leading to increased computing requirements.

PANDA will have a multitude of detector modules dedicated to the reconstruction of charged tracks. The algorithms discussed here focus specifically on the Straw Tube Tracker (STT), which is also highlighted in fig. 1. The STT consists of 4224 gas-filled tubes, arranged in a hexagonal layout. 19 layers of tubes are aligned in parallel to the beam direction, whereas the remaining 8 stereo layers have an inclination angle of $\pm 3^{\circ}$, allowing for longitudinal track

\footnotetext{
*e-mail: michael.papenbrock@physics.uu.se
} 

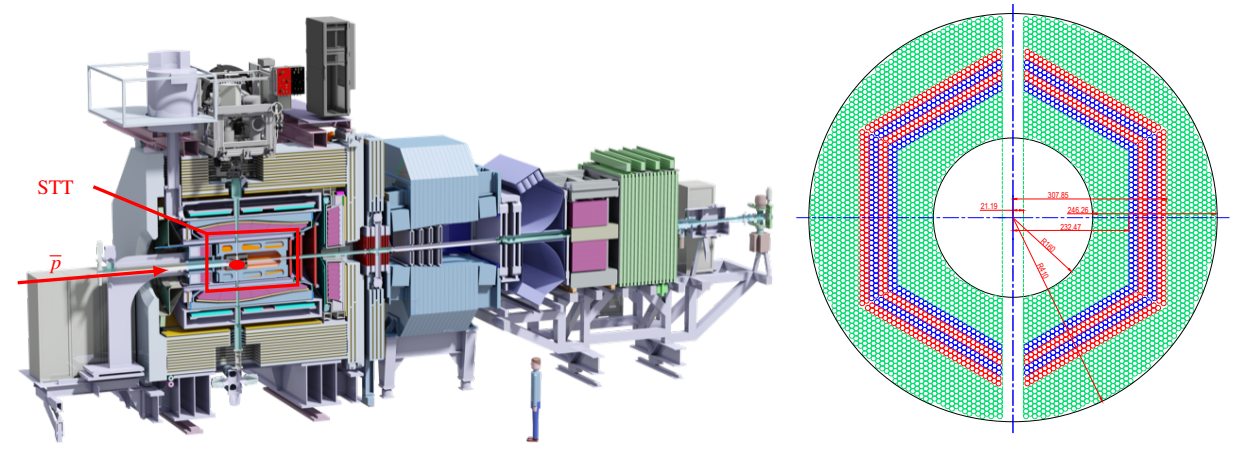

Figure 1: Left: The PANDA detector. Highlighted in red are the Straw Tube Tracker (STT), the direction of the incoming antiproton beam, and the interaction point. Right: A cross section of the STT. Tubes parallel to the beam direction are coloured green. Tubes inclined by $\pm 3^{\circ}$ are coloured in blue and red, respectively.

reconstruction. Each tube has a diameter of $10 \mathrm{~mm}$. When a charged particle traverses a tube, it creates an electron avalanche. The minimum distance of the particle trajectory from the centre wire can be derived from the minimum drift time of the electrons. Since the direction of the trajectory is not known hitherto, the possible position form a circle, also referred to as isochrone, with the minimum distance as its radius. The STT has an isochrone resolution of $150 \mu \mathrm{m}[2]$.

\section{Cellular Automaton}

Secondary tracks from displaced vertices add a significant challenge to the reconstruction, especially when the pattern recognition has to be carried out in real time. Many of the traditional algorithms can be adapted to cater for these. For example, a conformal mapping [3] with subsequent Hough transformation [4] can be performed after shifting the origin to another vertex position and detect the secondary tracks. To do conformal mapping for displaced tracks the pivot point shall be one of the hits. However, this comes at the cost of additional computations proportional to the number of vertex hypotheses. For online reconstruction, this might not always be affordable.

Cellular automata [5] are an interesting approach as they reduce the spatial information of the detector signals to neighbourhood relations between so-called cells. In the PANDA Straw Tube Tracker, a cell represents a single straw tube. Since the tubes parallel to the beam pipe are aligned in a hexagonal layout, each tube is surrounded by six neighbours ${ }^{1}$. Each cell can have one of two states: active or inactive. All cells together form a state vector. Using a cellular automaton as part of a track reconstruction algorithm differs from its common use, where typically new generations of the state vector are created based on a predefined set of rules referring to specific neighbourhood relations. In our case, all cells are marked as inactive in the beginning. Once a detector hit occurs, the corresponding cell is marked as active. The active cells are then classified as either unambiguous, when they have $\leq 2$ active neighbours, or ambiguous, when the number of active neighbours exceeds 2. Afterwards, unambiguous cells are easily combined into tracklets. Finally, preliminary track fits are performed to these

\footnotetext{
${ }^{1}$ Note that tubes on the edges of the detector as well as in the stereo layers are exempt from this consideration.
} 


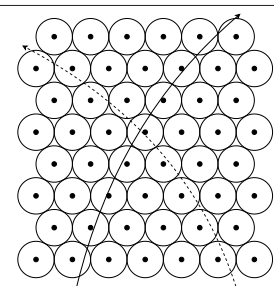

(a)

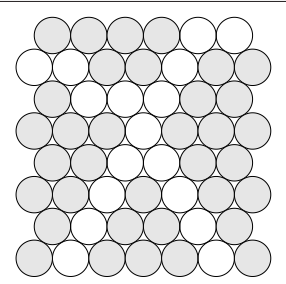

(b)

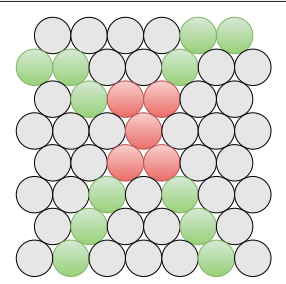

(c)

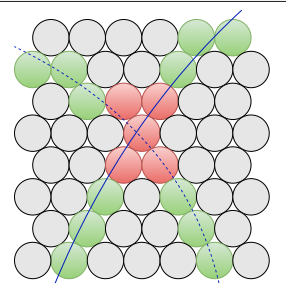

(d)

Figure 2: Illustration of the cellular automaton workflow. (a) Particles traverse the detector. (b) Cells corresponding to detector hit are marked as active. (c) Active cells are classified as unambiguous (green) or ambiguous (red). (d) Track fits are used to resolve ambiguities and merge tracklets.

tracklets in order to resolve ambiguities and merge disjoint tracklets originating from the same particle.

Earlier versions of the cellular automaton track finder for PANDA were utilising purely spatial information for clustering hits into tracklets [6] and later modified to be accelerated on GPUs [7]. However, due to PANDA's time-based approach to data acquisition (see section 1), the neighbourhood information of cells will be influenced by overlapping or adjacent events. An additional dimension had to be added to the pattern recognition: time [8]. The updated version of this track finder only accepts two hits as neighbours if their timing of their signals is no more than $250 \mathrm{~ns}$ apart. This time is based on the maximum drift time of the electrons in the straw tubes, including a margin for error.

Figure 3: Performance of different implementations of the cellular automaton track finder.

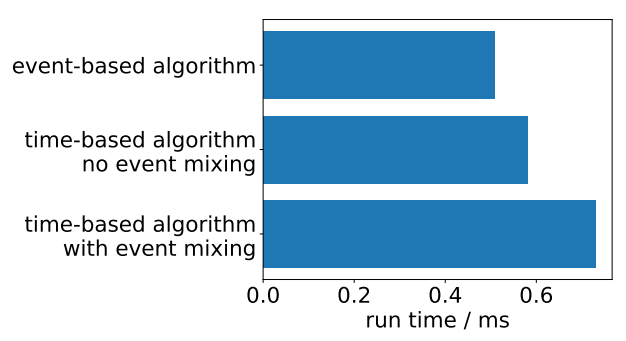

The computational performance of this track finder is illustrated in fig. 3. The algorithms were embedded in a full, serial event reconstruction, which took $\approx 10 \mathrm{~ms}$ to execute on an Intel i7 CPU with $3.4 \mathrm{GHz}$. The top row shows the run-time of the event-based algorithm. The middle row shows the time-based algorithm. However, the collision rate in the simulation was chosen to be 2 $\mathrm{MHz}$ (500 ns mean time between events), which is so low that event mixing is negligible. These conditions are similar to what is expected during the initial datataking phase of PANDA. The third row has a higher collision rate of $20 \mathrm{MHz}$ (50 ns mean time between events), where event mixing is common. It can be observed that the overall run-time of the algorithm is quite short when compared to the full event reconstruction time. The additional computational footprint of taking into account the temporal hit data is even smaller, adding $\approx 1-2 \%$ to the total reconstruction time, depending on the collision rate.

\section{Longitudinal track reconstruction}

With the majority of its tubes being aligned in parallel with the antiproton beam direction, the STT lends itself best to the reconstruction of transversal track components. However, 
included are also eight stereo layers with an inclination angle of $\pm 3^{\circ}$. For some track finders, these demand separate treatment. Consider for example the cellular automaton (see section 2): With a relative inclination angle between individual tubes, the neighbourhood relations become more complex and ambiguous. Similar considerations may apply for other track finders. To account for that, an independent algorithm was developed that extracts the hit locations from the stereo layers.

The procedure assumes that the transversal components of a track have already been determined using the straw tubes of the STT which are oriented parallel to the beam direction. Since this algorithm is intended to be used online during data taking, a simple circular track model is used. First, the isochrone radii in the $(S, z)$ space, with $z$ as the position along the beam direction and $S$ as the arc length along the track helix, are obtained from the stereo layers. However, the center positions of these isochrones have a large spatial ambiguity, since they can be anywhere along the respective tube wires. Aligning the isochrones to an existing transversal track fit reduces this to a left/right ambiguity, illustrated in fig. 4. Three different methods were developed to resolve this ambiguity, which will be explained in the following.

\subsection{Combinatorial path finder}

The combinatorial path finder [9] attempts to find the "straightest" path along all possible combinations of isochrone locations. In the first step, all possible connections between adjacent layers are determined as illustrated in fig. 5a. For all neighbouring lines, the angle between them is calculated. Unrealistic paths with $\theta<90^{\circ}$ are already rejected here (see fig. 5b). Finally, the path is selecting by minimising the cost function:

$$
w=\sum_{j}^{n}\left(180^{\circ}-\theta_{j}\right)^{2}
$$

A successful selection of isochrone positions is shown in fig. 5c.

\subsection{Hough transformation}

The Hough transformation [4] has long been a staple in track reconstruction, having been developed specifically for this purpose in bubble chamber experiments. For each hit, a set of all possible lines going through it are calculated. These are usually parameterised in terms of their inclination angle $\theta$ and distance from the origin $R$, which span the accumulator space. The accumulator space is filled with the parameters of every line. Maxima emerge when a set of parameters results in a line that goes through multiple hits. Since in this specific method only one correct path is expected per definition, the global maximum of the accumulator space yields directly the correct track parameters. 


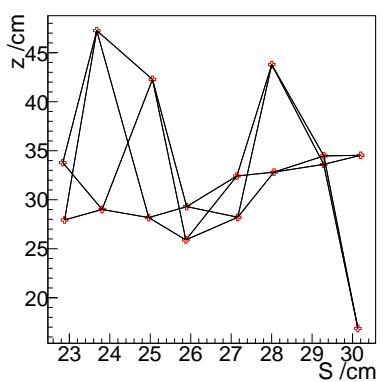

(a)

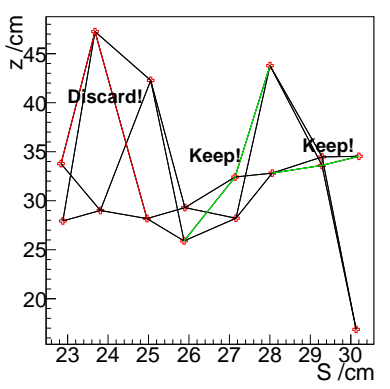

(b)

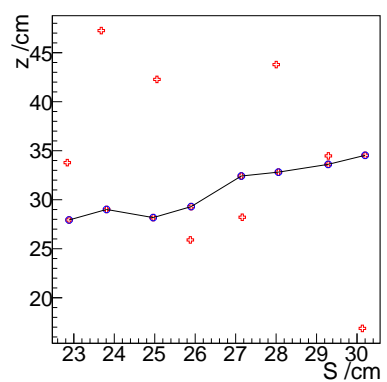

(c)

Figure 5: The isochrone center positions plotted in the $(S, z)$ space. Figure 5a shows all possible connections between adjacent stereo layers in the STT. In fig. 5b, connections with $\theta<90^{\circ}$ are rejected. Finally, fig. $5 \mathrm{c}$ shows the selected path after minimising the cost function eq. (1).

The optimal binning for $R$ and $\theta$, and therefore the amount of lines that have to be calculated, depends to some extent on the detector resolution. By varying the binning of these parameters we found that 25 bins for $R$ and 90 bins in $\theta$ resulted in the best compromise of efficiency and purity in the reconstruction.

\subsection{Recursive annealing fit}

The recursive annealing fit offers a straightforward and computationally efficient approach to the problem at hand. It capitalises on the fact that there are exactly two possible isochrone locations for each straw tube. In other words, exactly one point has to be rejected for each tube. In the first step, a line is fitted to all points. One by one, the point with the largest residual to the fit is removed until one point has been rejected for each tube. Since the fit is linear, the recalculation of the fit after each removal is computationally inexpensive.

\subsection{Comparison}

To investigate the performance of the three algorithms, several event samples were simulated. Each sample contained 10000 events comprised of 4 prompt muons, two of each charge, at muon momenta of $0.1,0.2,0.5,1,2$, and $5 \mathrm{GeV} / \mathrm{c}$, covering the full expected momentum range at PANDA as well as isotropically illuminating the STT. As performance observables, we define the efficiency as the ratio of correctly found hits and all hits that were generated, i.e.

$$
\text { efficiency }=\frac{N_{\text {correctly found hits }}}{N_{\text {all hits }}^{M C}},
$$

and the purity as the ratio of the correctly found hits and all hits that were associated with the final tracks, i.e.

$$
\text { purity }=\frac{N_{\text {correctly found hits }}}{N_{\text {all found hits }}} \text {. }
$$

The results have been compiled in fig. 6. While all algorithms produce good efficiencies, the Hough transformation yields notably lower purities. All algorithms struggle at lower momenta. However, the low momentum bins chosen here are just barely within the geometrical 

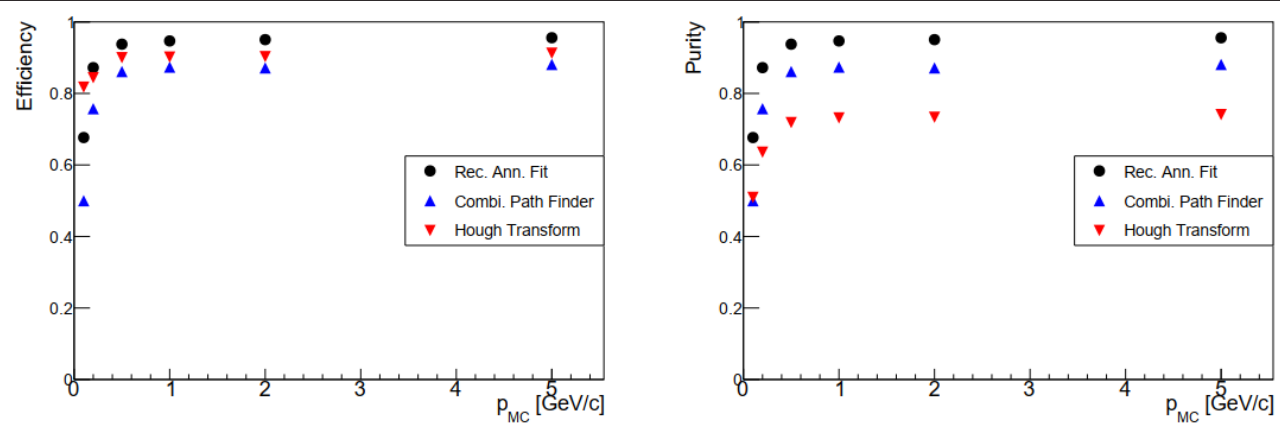

Figure 6: The efficiency and purity for the recursive annealing fit (black dots), the combinatorial path finder (blue triangles up), and the Hough transformation (red triangles down).

acceptance of the STT. For these tracks, energy loss plays a more important role so that a simple, circular track model proves to be insufficient.

Finally, it can be observed that the recursive annealing fit performs best in all categories and reaches efficiencies and purities of above $95 \%$.

\section{Conclusions}

Track and event reconstruction with PANDA will be a challenging task. The pure software trigger puts new demands on online reconstruction algorithms, which have to educate trigger decisions on free-streaming data. The work on a new generation of time-based reconstruction algorithms is underway and first developments using cellular automata look promising with very small additional computational costs. This approach is particularly interesting for tracks from displaced vertices as it makes few geometrical assumptions. The reconstruction of longitudinal momentum components with the PANDA Straw Tube Tracker was investigated using three different pattern recognition algorithms. Out of these, the algorithmically most straightforward one, i.e. the recursive annealing fit, showed the best performance with efficiencies and purities above 95\%. The developments shown here will be important pieces in the future reconstruction software of the PANDA experiment.

The authors are grateful to the Knut and Alice Wallenberg Foundation for the support.

\section{References}

[1] PANDA Collaboration, arXiv:0903.3905 (2009)

[2] PANDA Collaboration, Eur. Phys. J. A 49, 25 (2013)

[3] M. Hansroul et al., Nucl. Inst. Meth. Phys. Res A 270, 498-501 (1988)

[4] P. V. C. Hough, Conf. Proc. C590914, 554-558 (1959)

[5] S. Wolfram, Theory and applications of cellular automata: including selected papers 1983-1986, World scientific (1986)

[6] J. Schumann, Bachelor thesis, Jülich (2013)

[7] J. Schumann, Master thesis, Jülich (2015)

[8] J. Regina et al., Proceedings CTD/WIT (2019)

[9] W. Ikegami Andersson, J. Phys. Conf. Ser. 742, no. 1, 012009 (2016) 